\title{
Adenosine Deaminase Deficiency
}

\author{
DISAPPEARANCE OF ADENINE DEOXYNUCLEOTIDES \\ FROM A PATIENT'S ERYTHROCYTES \\ AFTER SUCCESSFUL MARROW TRANSPLANTATION
}

\author{
Shi-Han Chen, Hans D. Ochs, and C. Ronald Scott, Department of Pediatrics, \\ School of Medicine, University of Washington, Seattle, Washington 98195 \\ Eloise R. Giblett, Puget Sound Blood Center, Seattle, Washington 98104 \\ AUBREy J. TINGLE, Department of Pediatrics, Faculty of Medicine, University of British \\ Columbia, Vancouver, British Columbia, Canada
}

\begin{abstract}
A B S TRACT Accumulation of adenine deoxynucleotides (dATP and dADP) in the erythrocytes of a patient with adenosine deaminase (ADA) deficiency was confirmed. The patient, now 18 mo old, was treated with a bone marrow transplantation from his HLA identical sister at $7 \mathrm{mo}$ of age. Before and after the transplant, his erythrocyte and lymphocyte ADA activities, as well as his erythrocyte nucleotide profiles, were measured. $10 \mathrm{wk}$ after the marrow transplant, no ADA activity could be detected in his erythrocytes, whereas there was a mixture of donor and patient lymphocytes as measured by ADA assays and karyotyping. At the same time, both dATP and dADP had disappeared from his erythrocytes, which were entirely of patient origin. These findings indicate that partial engraftment of donor lymphocytes into an ADA-deficient patient is capable of "correcting" alterations of deoxynucleotide concentrations in the patient's ADA-deficient erythrocytes.
\end{abstract}

\section{INTRODUCTION}

Many cases of inherited deficiency of adenosine deaminase $(\mathrm{ADA})^{1}{ }^{1}$ EC 3.5.4.4.) associated with severe

\footnotetext{
Dr. Ochs is an investigator of the Howard Hughes Medical Institute.

Received for publication 7 August 1978 and in revised form 15 September 1978.

${ }^{1}$ Abbreviation used in this paper: ADA, adenosine deaminase.
}

combined immune deficiency have been described ( 1 , 2). Abnormal quantities of adenosine, adenine, ADP, ATP, and cyclic AMP have been reported in the serum, urine, erythrocytes, and lymphocytes of ADA-deficient patients (3-6). Recently, Cohen et al. (7) and Coleman et al. (8) independently found high levels of adenine deoxynucleotides (dATP and dADP) accumulated in the erythrocytes of two affected patients. Cohen et al. (7) suggested that a similar accumulation of dATP in the lymphoid tissues of ADA-deficient children may be one of the underlying causes of their immunodeficiency via DNA "starvation" through the inhibitory action of the dATP on ribonucleotide reductase. More recently, Donofrio et al. (9) have documented an accumulation of dATP in the lymphocytes of one of these patients. In this paper we confirm the accumulation of adenine deoxynucleotides (dATP and dADP) in erythrocytes of an ADA-deficient patient and report the eventual disappearance of the nucleotides from his own erythrocytes after a successful engraftment of donor lymphocytes.

\section{METHODS}

Patient. The male child was born to a Canadian couple of German descent in February 1977 after an uncomplicated pregnancy. At the age of $5 \mathrm{wk}$ he developed a persistent respiratory infection. On 1 June he was admitted to the Vancouver Children's Hospital for treatment of sepsis and severe pneumonia. No thymus was visible on $x$ ray. His leukocyte count was 11,200 with only $4 \%$ lymphocytes. His lympho- 
cyte cultures had a markedly impaired response to phytohemagglutinin, concanavalin $\mathbf{A}$, and pokeweed mitogen, suggesting a severe combined immunodeficiency syndrome. Subsequently, no ADA activity could be detected in his erythrocytes. The patient was admitted to the Fred Hutchinson Cancer Research Center in Seattle in August 1977, where he received a bone marrow transplant $\left(4 \times 10^{8}\right.$ cells $\left./ \mathrm{kg}\right)$ from his HLA-identical, mixed lymphocyte culture nonreactive, female sibling, who had normal erythrocyte ADA activity. She was the sole source of marrow and blood received by the patient. Her erythrocytes, unlike his, had the blood group antigen, N. Now, at 18 mo of age, the child is clinically and immunologically normal.

Chemicals. Chemicals were purchased from Sigma Chemical Company, St. Louis, Mo.; $\left[{ }^{3} \mathrm{H}\right]$ adenosine, $(6.4 \mathrm{Ci} /$ mmol) was obtained from Amersham Corp., Arlington Heights, Ill.

Cell separation and extract preparation. Fresh venous blood $(5 \mathrm{ml})$ was drawn into heparinized tubes. Lymphocytes were separated from erythrocytes and plasma by a FicollHypaque method (Ficoll, Pharmacia Fine Chemicals Inc., Piscataway, N. J.; Hypaque, Winthrop Laboratories, Sterling Drug Co., New York) (10). Hemolysates and leukocyte extracts were prepared as previously described (11). The fresh prepared extracts were used for ADA activity assays.

ADA activity assays. ADA was assayed spectrophotometrically (12) and also by an isotopic method. In the isotopic method, the reaction mixture contained $40 \mu \mathrm{l}$ of 30 $\mu \mathrm{M}\left[\mathrm{G}-{ }^{3} \mathrm{H}\right]$ adenosine $(250 \mathrm{mCi} / \mathrm{mmol})$ in phosphate buffer (pH 7.4). The reaction was initiated in duplicate by adding enzyme solution $(1-5 \mu \mathrm{l})$ and stopped at $0 \mathrm{~min}, 30 \mathrm{~min}$, and up to $2.5 \mathrm{~h}$ by immersing the tubes in boiling water for $1 \mathrm{~min}$. Aliquots $(10-20 \mu \mathrm{l})$ were spotted on a silica gel thin-layer chromatography plate (EM Laboratories, Inc., E. Merck, Darmstadt, Elmsford, N. Y.; F-254) for separation of adenosine, inosine, and hypoxanthine with a solvent that contained equal volumes of $94 \% n$-butanol and $44 \%$ aqueous propionic acid. The adenosine, inosine, and hypoxanthine areas were cut out, suspended in Aquasol (New England Nuclear, Boston, Mass.), and assayed for ${ }^{3} \mathrm{H}$ activity in a Packard Scintillation Counter (Packard Instrument Co., Downers Grove, Ill.) with

TABLE I

Erythrocyte and Lymphocyte ADA Activity in a Patient before and after Marrow Transplantation

\begin{tabular}{ccc}
\hline \multicolumn{1}{c}{ Subject } & Erythrocyte ADA & Lymphocyte ADA \\
\hline & $\mu$ mol/h/g $\mathrm{Hb}$ & $\mu$ mol/min/10 cells \\
Patient: & & \\
Pre-transplantation & $<0.2(3)^{*}$ & $<0.05(1)$ \\
Post-transplantation (wk) & & \\
3 & $2.7(3)$ & $0.8(1)$ \\
5 & $2.4(3)$ & - \\
6 & $1.5(2)$ & $1.0(3)$ \\
7 & $1.6(1)$ & $0.65(1)$ \\
9 & $0.7(1)$ & - \\
10 & $<0.2$ & - \\
12 & $<0.2$ & - \\
15 & $<0.2$ & $0.4(2)$ \\
20 & $<0.2$ & $0.3(1)$ \\
Normal controls & $36.1 \pm 6.7(67)$ & $1.2 \pm 0.3(5)$ \\
\hline
\end{tabular}

$\mathrm{Hb}$; hemoglobin.

* ( ) Number of independent determinations. counting efficiency of $49 \%$. ADA activity was calculated from the quantity of inosine and hypoxanthine produced.

High-performance liquid chromatography for nucleotide assay. Perchloric acid extracts of erythrocytes were prepared by the method described by Beutler (13). The neutralized extracts were used immediately for experiments or stored in $-20^{\circ} \mathrm{C}$ for up to 1 mo without loss of the nucleotide content. Nucleotides were resolved by high-performance liquid chromatography. The perchloric acid extracts $(20-80 \mu \mathrm{l})$ were injected into a Waters $\mu$-Bondapak $\mathrm{NH}_{2}, 4-\mathrm{mm} \times 30-\mathrm{cm}$ column (Waters Associates, Inc., Milford, Mass.) equilibrated

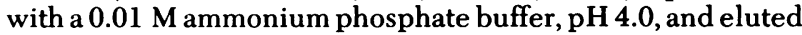
with a linear gradient of $0.125 \mathrm{M}$ ammonium phosphate buf-

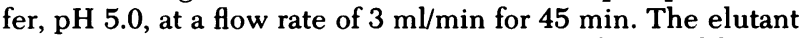
was monitored at $254 \mathrm{~nm}$. The peaks were identified by cochromatography of the known standards in the column and by a thin-layer chromatography method (14). The areas under the peaks were measured by an Autolab IV integrator (Autolab Div., Spectra-Physics, Santa Clara, Calif.). The concentrations
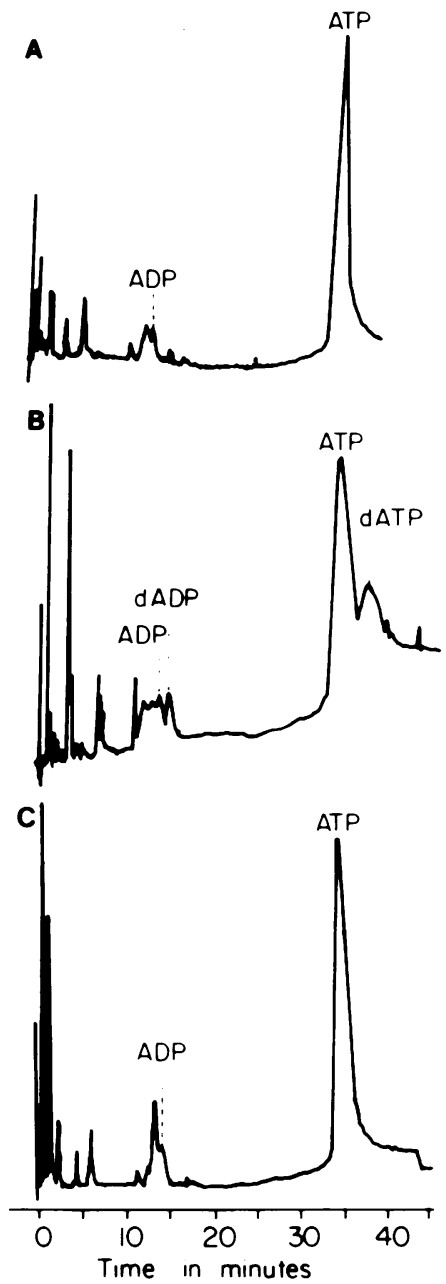

FIGURE 1 High-performance liquid chromatographs show separation of some adenine nucleotides (ATP and ADP) and deoxynucleotides (dATP and dADP) in erythrocyte extracts of an ADA-deficient patient. (A) normal control; (B) ADAdeficient patient before a marrow transplantation; $(C)$ the same patient $10 \mathrm{wk}$ after the transplantation. 
TABLE II

Adenine Nucleotides and Adenine Deoxynucleotides in the Patient's Erythrocytes before and after Transplantation

\begin{tabular}{|c|c|c|c|c|c|}
\hline Subject & ADP & ATP & dADP & dATP & dATP/ATP \\
\hline & \multicolumn{5}{|c|}{$\mu \mathrm{mol} / \mathrm{ml}$ erythrocytes } \\
\hline \multicolumn{6}{|l|}{ Patient } \\
\hline \multicolumn{6}{|c|}{ Pre-transplantation (wk) } \\
\hline 4 & 0.12 & 1.2 & 0.16 & 0.35 & 0.3 \\
\hline 3 & 0.16 & 0.43 & 0.16 & 0.57 & 1.3 \\
\hline 0 & 0.1 & 1.2 & 0.1 & 0.2 & 0.17 \\
\hline \multicolumn{6}{|c|}{ Post-transplantation (wk) } \\
\hline 9 & 0.27 & 1.9 & $<0.01$ & $<0.01$ & \\
\hline 10 & 0.18 & 1.7 & $<0.01$ & $<0.01$ & \\
\hline 15 & 0.22 & 1.2 & $<0.01$ & $<0.01$ & \\
\hline 20 & 0.2 & 1.2 & $<0.01$ & $<0.01$ & \\
\hline Patient's mother (2) & 0.12 & 1.1 & $<0.01$ & $<0.01$ & \\
\hline Normal control (17) & $0.2 \pm 0.05$ & $1.3 \pm 0.2$ & $<0.01$ & $<0.01$ & \\
\hline
\end{tabular}

of the individual nucleotides were calculated with known nucleotides as standards.

\section{RESULTS}

Table I shows the patient's erythrocyte and lymphocyte ADA activities before and after the transplantation. Before treatment, there was no detectable erythrocyte ADA ( $<0.2 \mathrm{U} / \mathrm{g}$ hemoglobin). $3 \mathrm{wk}$ after the transplant, $2.7 \mathrm{U} / \mathrm{g}$ hemoglobin (about $7 \%$ of normal) was detected, a value in accord with the number of $\mathrm{N}$-positive erythrocytes then present in the N-negative recipient's blood. After the 10th wk, neither ADA nor N-positive erythrocytes were detectable. On the other hand, the enzyme specific activity in the lymphocytes was nearly normal during the 3-7-wk period after transplantation. Later, at $15-20 \mathrm{wk}$, the values were $30-35 \%$ of normal.

Fig. 1 shows high-performance liquid chromatographs of erythrocyte nucleotide profiles of (A), a normal control; (B), the patient $4 \mathrm{wk}$ before transplantation; and $(\mathrm{C})$, the patient $10 \mathrm{wk}$ after transplantation. The major peaks of the normal control were identified as ADP and ATP in the chromatograph. The chromatograph of the patient's pretransplant extract revealed a strikingly different pattern, with one large peak after the ADP peak and another peak after ATP. Neither of these two additional peaks was seen in the erythrocyte chromatographs of 17 normal controls or in those of the patient's parents. The new peaks co-chromatographed in the high-performance liquid chromatography column with standard dADP and dATP, respectively. The identification of dATP was also confirmed by co-chromatography of the eluted compound with the standard dATP on a thin-layer chromatograph. The dADP and dATP peaks were not present in chromatographs of the patient erythrocytes 10 wk after marrow transplantation, although no erythrocyte ADA was detectable. At that time, his immunologic functions showed marked improvement as indicated by in vitro lymphocyte transformation to mitogens (phytohemagglutinin, concanavalin $\mathrm{A}$, and pokeweed mitogen) and in vivo antibody responses to bacteriophage $\phi X 174)$. The levels of dADP, dATP, ADP, and ATP in the patient's erythrocytes are summarized in Table II. dADP or dATP was undetectable $(<0.01 \mathrm{mM})$ in normal erythrocytes, whereas both were present in significant quantities in those of the patient. The dATP concentrations were $0.35,0.57$, and $0.2 \mathrm{mM}$, respectively, in three different blood samples before the transplantation. The ratio of dATP:ATP on the samples were $0.3,1.3$, and 0.17 , respectively. The dADP levels were from 0.10 to $0.16 \mathrm{mM}$, which was about the same as the ADP level found in his erythrocytes.

\section{DISCUSSION}

3 wk after receiving a bone marrow transplant from his normal sister, the patient's erythrocyte ADA activity rose to $7 \%$ of normal and then gradually declined, becoming undetectable $10 \mathrm{wk}$ after treatment. Conversely, the patient's lymphocyte $\mathrm{ADA}$ activity rose to a nearly normal level and then fluctuated moderately during the 20 wk after transplantation. $8 \mathrm{mo}$ after transplantation, karyotype analysis of his peripheral lymphocytes with phytohemagglutinin stimulation showed $67 \% \mathrm{XX}$ and $33 \% \mathrm{XY}$ cells.

The finding of large quantities of adenine deoxynucleotides in the patient's erythrocytes was in accord with two previous reports $(7,8)$. By following the nucleotide profiles, we showed that the erythrocyte adenine deoxynucleotides in our patient disappeared after transplantation, even though these cells were of patient origin, as revealed by absence of both ADA and the $\mathrm{N}$ antigen. Therefore, a process capable of removing accumulated adenine deoxynucleotides from these erythrocytes must have developed after treatment. 
There is evidence (15) that in normal human erythrocytes deamination of deoxyadenosine is about 75-180fold greater than its phosphorylation. As a consequence, the deoxyadenosine of normal erythrocytes is mainly metabolized to deoxyinosine and then to hypoxanthine. However, in ADA-deficient patients, deoxyadenosine is converted to deoxy-AMP and finally accumulates in the form of dADP and dATP. Even though a "take" of the donor's erythrocytes did not occur, lymphocyte engraftment took place, permitting deamination of deoxyadenosine accumulated in the patient's other cells, including erythrocytes and probably their precursor cells. Furthermore, this deaminating capability of the grafted lymphocytes apparently permits the appearance of some of the patient's ADA-deficient lymphocytes, thus achieving a lymphocyte chimerism of ADA normal and abnormal cells.

The mechanism by which accumulated deoxyadenosine and a consequent rise in DADP and dATP causes lymphocyte dysfunction is still a matter of speculation. Currently, the most attractive view is that dATP has an inhibiting effect on ribonucleotide reductase and decreases the availability of other deoxynucleotides for DNA synthesis in lymphocytes. The study of this patient establishes that the successful engraftment of donor lymphocytes in an ADA-deficient patient reduces the concentration of dATP and dADP to normal values within the patient's erythrocytes and also allows a portion of the patient's lymphocytes to survive. These observations are compatible with the concept that one major toxic factor in ADA deficiency is an accumulation of dATP.

\section{ACKNOWLEDGMENTS}

We thank Dr. Holger Hoehn, Department of Pathology, University of Washington, for karyotyping of the patient lymphocytes, and the physicians of the Fred Hutchinson Cancer Research Center for their clinical supervision of the bone marrow transplant.

The work was supported in part by U. S. Public Health Service grants AI 12617, AI 07073, GM 15253, and HL 17265, and by grants from the National Foundation-March of Dimes. A portion of this work was also conducted through the Clinical Research Center Facility of the University of Washington with the support of a grant (RR-37) from the National Institute of Health.

\section{REFERENCES}

1. Giblett, E. R., J. E. Anderson, F. Cohen, B. Pollara, and H. J. Meuwissen. 1972. Adenosine deaminase deficiency in two patients with severely impaired cellular immunity. Lancet. II: 1067-1069.

2. Meuwissen, H. J., R. J. Pickering, B. Pollara, and Z. H. Porter. 1975. Combined Immunodeficiency Disease and Adenosine Deaminase Deficiency. Academic Press, Inc., New York.

3. Mills, G. C., F. C. Schmalstig, K. B. Trimmer, A. S. Goldman, and R. M. Goldblum. 1976. Purine metabolism in adenosine deaminase deficiency. Proc. Natl. Acad. Sci. U. S. A. 23: 2867-2871.

4. Agarwal, R. P., G. W. Crabtree, R. E. Parks, Jr., J. A. Nelson, R. Keightly, R. Parkman, F. S. Rosen, R. C. Stern, and S. H. Polmar. 1976. Purine nucleoside metabolism in the erythrocytes of patients with adenosine deaminase deficiency and severe combined immunodeficiency. $J$. Clin. Invest. 57: 1025-1035.

5. Schmalstig, F. C., J. A. Nelson, G. C. Mills, T. M. Monahan, A. S. Goldman, and R. M. Goldblum. 1977. Increased purine nucleotides in adenosine deaminase-deficient lymphocytes. J. Pediatr. 41: 48-51.

6. Snyder, F. F., J. Mendelsohn, and J. E. Seegmiller. 1976. Adenosine metabolism in phytohemagglutinin-stimulated human lymphocytes. J. Clin. Invest. 58: 654-666.

7. Cohen, A., R. Hirschhorn, S. D. Horowitz, A. Rubenstein, S. H. Polmar, R. Hong, and D. W. Martin. 1978. Deoxyadenosine triphosphate as a potentially toxic metabolite in adenosine deaminase deficiency. Proc. Natl. Acad. Sci. U. S. A. 75: 472-476.

8. Coleman, M. S., J. Donofrio, J. J. Hutton, L. Hahn, A. Daoud, B. Lampkin, and J. Dyminski. 1978. Identification and quantitation of adenine deoxynucleotides in erythrocytes of a patient with adenosine deaminase deficiency and severe combined immunodeficiency.J. Biol. Chem. 253: 1619-1626.

9. Donofrio, J., M. S. Coleman, J. J. Hutton, A. Daoud, B. Lampkin, and J. Dyminski. 1978. Overproduction of adenine deoxynucleosides and deoxynucleotides in adenosine deaminase deficiency with severe combined immunodeficiency disease. J. Clin. Invest. 62: 884-887.

10. Boyum, A. 1974. Separation of blood leukocytes and lymphocytes. Tissue Antigens. 4: 269-274.

11. Chen, S. H., L. A. Malcolm, A. Yoshida, and E. R. Giblett. 1971. Phosphoglycerate kinase: An X-linked polymorphism in man. Am. J. Hum. Genet. 23: 87-91.

12. Scott, C. R., S. H. Chen, and E. R. Giblett. 1974. Detection of the carrier state in combined immunodeficiency disease associated with adenosine deaminase deficiency. J. Clin. Invest. 53: 1194-1196.

13. Beutler, E. 1975. Red Cell Metabolism: A Manual of Biochemical Methods. Grune \& Stratton, Inc., New York. 16-17.

14. Randerath, K. 1963. Separation of deoxyribonucleotides from ribonucleotides by anion-exchange thin-layer chromatography. Biochim. Biophys. Acta. 76: 622-624.

15. Snyder, F. F., and J. F. Henderson. 1973. Alternative pathways of deoxyadenosine and adenosine metabolism. $J$. Biol. Chem. 248: 5899-5904. 